Research Paper

\title{
CD I 4 Targets Complement Receptor 3 to Lipid Rafts during Phagocytosis of Borrelia burgdorferi
}

\author{
Kelly L. Hawley 1, Itziar Martín-Ruiz 2, Juan M. Iglesias-Pedraz 1, Brent Berwin 3, and Juan Anguita 1,2,4凶 \\ 1. Department of Veterinary and Animal Sciences, University of Massachusetts at Amherst, Amherst, MA 01003, USA. \\ 2. Proteomics Unit, CIC bioGUNE. 48160 Derio, Bizkaia, Spain. \\ 3. Department of Microbiology and Immunology. Dartmouth College of Medical Center. Lebanon, NH 03576, USA. \\ 4. Ikerbasque, Basque Foundation for Science, 48003 Bilbao, Bizkaia, Spain.
}

$\triangle$ Corresponding author: Juan Anguita, PhD, Proteomics Unit. CIC bioGUNE. Technology Park of Bizkaia. 48160 Derio, Bizkaia, Spain. Phone: +34 944061 311; Fax: +34 944061 301; e-mail: janguita@cicbiogune.es.

(c) Ivyspring International Publisher. This is an open-access article distributed under the terms of the Creative Commons License (http://creativecommons.org/ licenses/by-nc-nd/3.0/). Reproduction is permitted for personal, noncommercial use, provided that the article is in whole, unmodified, and properly cited.

Received: 2013.07.10; Accepted: 2013.08.03; Published: 2013.08.20

\begin{abstract}
Phagocytosis of Borrelia burgdorferi, the causative agent of Lyme disease, is mediated partly by the interaction of the spirochete with Complement Receptor (CR) 3. CR3 requires the GPI-anchored protein, CDI4, in order to efficiently internalize CR3-B. burgdorferi complexes. GPI-anchored proteins reside in cholesterol-rich membrane microdomains, and through its interaction with partner proteins, help initiate signaling cascades. Here, we investigated the role of CDI4 on the internalization of $B$. burgdorferi mediated by CR3. We show that CR3 partly colocalizes with CDI4 in lipid rafts. The use of the cholesterol-sequestering compound methyl- $\beta$-cyclodextran completely prevents the internalization of the spirochete in CHO cells that co-express CDI 4 and CR3, while no effect was observed in CDI Ib-deficient macrophages. These results show that lipid rafts are required for CR3-dependent, but not independent, phagocytosis of B. burgdorferi. Our results also suggest that $\mathrm{CDI} 4$ interacts with the C-lectin domain of $\mathrm{CR} 3$, favoring the formation of multi-complexes that allow their internalization, and the use of $\beta$-glucan, a known ligand for the C-lectin domain of CR3, can compensate for the lack of CDI4 in CHO cells that express CR3. These results provide evidence to understand the mechanisms that govern the interaction between CR3 and CDI4 during the phagocytosis of B. burgdorferi.
\end{abstract}

Key words:

\section{Introduction}

Phagocytosis is an important component of innate immunity that leads to the destruction of pathogens, the production of proinflammatory factors, and the initiation of acquired immune responses through antigen presentation [1]. During infection with Borrelia burgdorferi, the causative agent of Lyme disease, phagocytosis is also a significant element: besides its role in the control of spirochetal burdens during infection, it is required for the induction of a full-blown proinflammatory response in both human monocytes and murine macrophages [2-5]. Phagocytosis of $B$. burgdorferi enhances, modulates and alters the proin- flammatory response of macrophages compared to the use of isolated pathogen-associated molecular patterns (PAMPs) [2, 5-8]. Because of its importance in the overall phagocytic cell response to the spirochete, it is imperative to clarify the mechanisms of internalization of the bacterium.

Phagocytosis of B. burgdorferi occurs through at least two mechanisms that either require signals emanating, or are independent from the adaptor molecule, MyD88. MyD88-dependent phagocytosis occurs through a yet to be identified receptor [3, 9-11], while phagocytosis mediated by CR3 $\left(\alpha_{\mathrm{M}} \beta_{2}, \mathrm{CD} 11 \mathrm{~b} / \mathrm{CD} 18\right)$ 
does not involve MyD88-derived signals [8]. We have previously demonstrated that the internalization of the spirochete mediated by CR3 requires the participation of the GPI-anchored protein co-receptor, CD14, which is not involved in the binding of the spirochete [8]. However, the role of CD14 during the internalization of B. burgdorferi remains undefined. Our results also showed that CR3-mediated phagocytosis modulates the inflammatory output of macrophages and its absence results in increased cardiac inflammation upon infection with the spirochete [8].

The $\alpha$ chain of integrins is responsible for the binding of specific ligands that occurs at the I-domain present on the $\mathrm{N}$-terminal region of the protein [12]. Some integrins, including CR3, also contain a C-lectin domain that has affinity for a diversity of sugars, including $\beta$-glucans [13]. The C-lectin domain is also responsible for their interaction with GPI-anchored proteins, including CD14 [13]. In several instances, CR3 alone is sufficient to mediate the internalization of microorganisms [14], while it can also require the participation of co-receptors to be able to initiate phagocytosis [15]. CD14 does not participate in the binding to the spirochete [8], and thus, is likely to allow the CR3-B. burgdorferi complexes to converge on the cellular surface and initiate the outside-in signals required for their internalization.

GPI-anchored proteins, lacking the capacity to signal to the cell by themselves, serve as co-receptors to a variety of cell surface molecules. These include some TLRs, as well as integrins [16, 17]. In most cases, the activity of GPI-anchor proteins facilitate the formation of cross-linked complexes, which can initiate the signals specific to the main receptor. Thus, upon LPS engagement, CD14 facilitates the translocation of TLR4 to membrane cholesterol-rich microdomains, wherefrom signals can emanate [17]. Another GPI-anchored protein, CD87, has been shown to trigger the location of integrins, such as CR3 to lipid rafts [16]. Therefore, the formation of these membrane microdomains is a characteristic of receptor-mediated signaling events that use these proteins as co-receptors.

In this report, we show that CD14 mediates the translocation of CR3 to cholesterol-rich microdomains in the plasma membrane, an event necessary for the phagocytosis of B. burgdorferi mediated by the integrin. The role of CD14 is probably also played by other GPI-anchored proteins, since the absence of this protein results in a partial, albeit consistent, reduction in the internalization of the spirochete. Furthermore, we show that CR3 crosslinking can occur in the presence of $\beta$-glucan that binds the C-lectin domain of the integrin, suggesting that the role of CD14 is circumscribed to its ability to interact with CR3 and promote its translocation to lipid rafts. These results clarify the molecular mechanisms that govern the phagocytosis of B. burgdorferi.

\section{Materials and Methods}

Mice. C57Bl/6 (B6), CD14- and CD11b-deficient mice were purchased from Jackson Laboratories (Bar Harbor, ME). The Institutional Animal Care and Use Committee at UMass Amherst approved all procedures involving animals.

Cells. Bone marrow-derived macrophages were generated as described [4]. Briefly, bone marrow cells were collected from the femoral shafts and cultured in 20\% L929-conditioned RPMI 1640 supplemented with $10 \%$ FCS (Hyclone Thermo Scientific, Waltham, MA), $2.4 \mathrm{mM}$ L-glutamine and 10\% penicillin-streptomycin (Invitrogen, Carlsbad, CA) in $100 \mathrm{~mm} \times 15 \mathrm{~mm}$ petri dishes (Fisher Scientific, Pittsburgh, PA) for 8 days at $37{ }^{\circ} \mathrm{C}$ with $5 \% \mathrm{CO}_{2}$. Non-adherent cells were then eliminated and adherent macrophages were scraped, counted and resuspended in serum-free RPMI medium $2 \mathrm{~h}$ prior to use.

CHO cells transfected with human CR3, or CR3 + CD14 [8] were maintained in Ham's F-12 medium (Sigma Chemical Co., St. Louis, MO). The macrophage-like cell line RAW264.7 was maintained in RPMI 1640.

Bacteria. The clone Bb914, a virulent strain 297 B. burgdorferi derivative, which contains a constitutively expressed $g f p$ reporter stably inserted into cp26 [18] was used throughout.

Phagocytosis assay. Phagocytosis of B. burgdorferi was analyzed as described $[4,8]$. The cells $\left(10^{6} / \mathrm{ml}\right)$ were cultured in serum- and antibiotic-free medium with $B$. burgdorferi at different multiplicity of infections (m.o.i.) for $4 \mathrm{~h}$, except where indicated. In some instances, the cells were co-incubated with $1 \mathrm{mM}$ $\beta$-glucan of different origins (barley, laminarin or $S$. cerevisiae, Sigma Chemical Co.). The cells were washed extensively and resuspended in PBS supplemented with 1\% FCS and analyzed by flow cytometry using an LSR II flow cytometer (BD Biosciences) or were further prepared for microscopic analysis. The data were analyzed with FlowJo for Mac, version 8.6 (Tree Star, Inc., Ashland, OR).

Inhibitor treatments. RAW264.7 and CHO-CR3/CD14 cells $\left(10^{6} / \mathrm{ml}\right)$ were preincubated for $30 \mathrm{~min}$ with 1 or $5 \mathrm{mM}$ of methyl- $\beta$-cyclodextran (Sigma Aldrich) at $37^{\circ} \mathrm{C}$ in serum- and antibiotic-free medium, followed by incubation with B. burgdorferi (m.o.i. 25) for 4 hours.

GPI-anchored protein shedding. RAW264.7 cells $\left(10^{6} / \mathrm{ml}\right)$ were preincubated for $20 \mathrm{~min}$ with $0.5-1$ units of phosphatidylinositol-specific phospholipase $\mathrm{C}$ (PI-PLC) from Bacillus cereus (Invitrogen) at $4{ }^{\circ} \mathrm{C}$, 
followed by extensive washing and incubation with Bb914 at an m.o.i. $=25$. Following the phagocytosis assay, spirochetal uptake was measured by flow cytometry, as described above. The extent of CD14 cleavage was determined by staining with a phycoerythrin-conjugated anti-CD14 mAb (BD Biosciences), followed by flow cytometry analysis.

Microscopy. To visualize internalized $B$. burgdorferi, the cells were washed and fixed in 3.7\% paraformaldehyde for 7 minutes and permeabilized with $0.1 \%$ Triton-X-100 for 5 min. Following blocking of non-specific binding with 5\% BSA for 60 minutes, the cells were stained with rhodamine phalloidin (Invitrogen) to visualize the actin cytoskeleton for 20 minutes at room temperature. The cells were mounted with Prolong Gold Anti-fade mounting reagent (Invitrogen). Photomicrographs were taken using a Zeiss Axiovert 200M inverted microscope (Thornwood, NY) equipped with Apotome and a Hamamatsu Orca camera (Bridgewater, NJ).

In order to assess the localization of CD11b to lipid rafts, $5 \times 10^{4}$ RAW 264.7 cells were incubated with Bb914 (m.o.i 25) for $1 \mathrm{~h}$. The cells were incubated with the subunit B of cholera toxin (CTxB) labeled with alexa fluor 594 (Invitrogen) the last $20 \mathrm{~min}$ of the incubation period, washed and fixed. The preparations were then incubated with anti-CD11b conjugated to allophycocyanin (APC, clone M1/70, BD Biosciences) $2 \mathrm{~h}$ at $\mathrm{RT}$ in the dark. Upon extensive washing, the slides were mounted and analyzed in a Zeiss LSM 510 Meta Confocal System.

Cell fractionation by sucrose-gradient ultracentrifugation. To determine whether CD11b colocalizes to lipid rafts, we performed a detergent extraction method followed by sucrose gradient centrifugation [19], with a few modifications. Briefly, $1 x$ $10^{7}$ RAW 264.7 cells were stimulated with B. burgdorferi (m.o.i. 25) or left unstimulated for $1 \mathrm{~h}$. The cells were washed, resuspended in $200 \mu \mathrm{l}$ of ice-cold lysis buffer (25 mM MES, pH 6.5, $150 \mathrm{mM} \mathrm{NaCl}, 1 \%$ Triton X-100, $1 \mathrm{mM}$ PMSF, and a protein inhibitor cocktail (Sigma Chemical Co.), and disrupted by passage through a 25G syringe. The cell lysate was mixed with an equal volume of $80 \%$ sucrose prepared in lysis buffer without TX-100, placed at the bottom of a discontinuous gradient $(650 \mu \mathrm{l}$ of $30 \%$ sucrose, $250 \mu \mathrm{l}$ of $5 \%$ sucrose) and centrifuged at 200,000 $\mathrm{xg}$ for $20 \mathrm{~h}$ at 4 ${ }^{\circ} \mathrm{C}$. Ten fractions were recovered from the top and precipitated with trichloroacetic acid. The samples were separated in a $10 \%$ polyacrylamide gel, transferred to nitrocellulose membranes and immunoblotted with anti-CD11b (M-19) and anti-CD14 (M-305) antibodies (Santa Cruz Biotechnology, Dallas, TX). The blots were also immunostained with anti-flotillin 2 and -actin antibodies (Sant Cruz Biotechnology) to determine the fractions belonging to lipid rafts.

Statistical Analysis. The results are presented as means \pm SE. Significant differences between means were calculated with the Student's $t$ test. $P$ values of 0.05 or less were considered statistically significant.

\section{Results}

CR3-and CD14 co-localize to cholesterol-rich microdomains during the phagocytosis of $B$. burgdorferi. CD14 resides in cholesterol-rich domains or lipid rafts where it recruits surface receptors allowing the induction of signaling cascades [20-22]. To determine whether CD14 and CR3 co-localize to lipid rafts during the interaction of macrophages with $B$. burgdorferi, we performed sucrose gradient assays in RAW264.7 cells that had been stimulated with $B$. burgdorferi (m.o.i. 25) or left unstimulated. CD11b co-localized with CD14 in lipid raft fractions (determined by the presence of flotillin 2, not shown) regardless of the activation status of the cells (Fig. 1A). We also analyzed the colocalization of lipid rafts during the phagocytosis of B. burgdorferi by RAW264.7 cells using confocal microscopy. The orthogonal projection of the cells showed instances in which cholera toxin subunit $\mathrm{B}(\mathrm{CT} \times \mathrm{B})$ colocalized with $B$. burgdorferi that had been internalized (Figure 1B, central panel), indicating association with lipid rafts. In other cases, the spirochete was not obviously associated with cholesterol-rich microdomains (Figure 1B, left and right panels). The analysis of $\mathrm{CD} 11 \mathrm{~b}$ and $\mathrm{CT} \times \mathrm{B}$ staining by confocal microscopy in the presence and absence of the spirochete also showed that CD11b colocalized with $\mathrm{CT \times B}$ in both unstimulated and $B$. burgdorferi-stimulated RAW264.7 cells (Fig. 1C). The attachment of $B$. burgdorferi to the surface of RAW264.7 cells occurred at least partly, in lipid rafts that also contained CD11b (Fig. 1C). These results showed that the phagocytosis of B. burgdorferi involved, to some extent, their association with cholesterol-rich membrane microdomains.

Lipid rafts are required for CR3-dependent, but not CR3-independent, phagocytosis of $B$. burgdorferi. To determine the contribution of lipid rafts to the phagocytosis of B. burgdorferi, we analyzed the internalization of the spirochete in the presence of the cholesterol-sequestering compound, methyl- $\beta$-cyclodextran (M $\beta C D)$. RAW264.7 cells treated with $1 \mathrm{mM} \mathrm{M \beta CD}$ showed, as expected [23], a reduction in the FcR-mediated internalization upon crosslinking with anti-CD16/CD32 Abs (Figure 2A). Similarly, a partial reduction in the capacity of RAW264.7 cells to internalize B. burgdorferi was observed at this concentration (Figure 2A). We further analyzed the role of lipid rafts on the phagocytosis of $B$. burgdorferi by flow cytometry. The use of $5 \mathrm{mM} \mathrm{M} \beta C D$ resulted 
in the reduction of phagocytosis by both RAW264.7 cells (Figure 2B) and BMMs (Figure 2C). Interestingly, while no effect was observed in BMMs that lacked CR3 (Figure 2D), cholesterol sequestration prior to the phagocytosis assay resulted in the almost complete abrogation of $B$. burgdorferi phagocytosis by CHO-CR3/CD14 cells (Figure 2E). These results indicated that CR3-dependent, but not independent, phagocytosis of B. burgdorferi depends on the presence of cholesterol-rich membrane microdomains.

CD14 deficiency results in a marginal reduction of B. burgdorferi phagocytosis. Our results show that CD14 cooperates with CR3 during the phagocytosis of $B$. burgdorferi likely by aiding in the formation of B. burgdorferi-CR3 complexes at the lipid raft fraction of the plasma membrane. Previous studies have suggested that a deficiency in CD14 does not affect the internalization of the spirochete by BMMs [20]. We further explored the role of CD14 by first subjecting RAW264.7 cells to a treatment with the enzyme phospholipase C from Bacillus cereus (PI-PLC). The treatment with PI-PLC for 20 minutes resulted in the reduction, but no abrogation, of CD14 surface expression in these cells (Figure 3A, top panel) and no effect was observed in their capacity to phagocytose $B$. burgdorferi (Figure 3A, bottom panel). We argued that since the colocalization of CD14 with CR3 is limited (Figure 1A), even at low doses of surface CD14, phagocytosis can occur with no impediment. To further analyze the role of CD14, we obtained BMMs from CD14-deficient mice and assayed their ability to internalize B. burgdorferi at low (10) and moderate (25) m.o.i. At an m.o.i $=10$, no effect was observed in the capacity of CD14-deficient BMMs to phagocytose the spirochete (Figure 3B, top panel), while some reduction was observed at the higher m.o.i (Figure 3B, bottom panel). Thus, these results suggest that low levels of CD14 are required to aid CR3 in the uptake of $B$. burgdorferi and in its absence, an effect is mostly evident at higher multiplicities of infection.


Fig I. CR3-mediated phagocytosis of B. burgdorferi requires the translocation of the integrin to lipid rafts. (A) RAW264.7 cells were incubated for I $\mathrm{h}$ with B. burgdorferi $($ m.o.i. $=25)$, lysed and layered onto a sucrose gradient. The gradients were separated by ultracentrifugation for $20 \mathrm{~h}$. Ten fractions were immunoblotted with anti-CDI Ib and anti-CDI4 Abs. Fraction numbers from the top are indicated. T: whole lysates ( $25 \mu \mathrm{g})$. (B) RAW cells were incubated with Bb9l4 for I $\mathrm{h}$ in the presence of CTXB for the last $20 \mathrm{~min}$. The cells were fixed and images obtained by confocal microscopy. The images represent orthogonal projections showing colozalization or lack thereof between Bb9/4 (green) and CTxB (red). (C) Confocal micrographs showing CDI Ib localization to lipid rafts. RAW264.7 cells were incubated with Bb9/4 (lower panels) or left unstimulated (upper panels) for 30 min. The cells were washed, fixed and stained with an anti-CDI Ib Ab (blue) and CTxB (red). Colocalization of CDI Ib and CTxB staining is indicated by the purple color in the small panel. The results presented are representative of 3-5 individual experiments. 


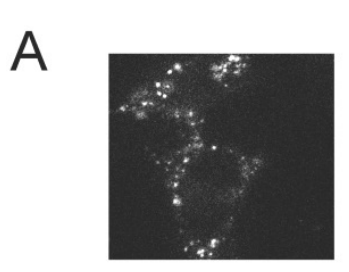

$$
+\mathrm{M} \beta \mathrm{CD}
$$
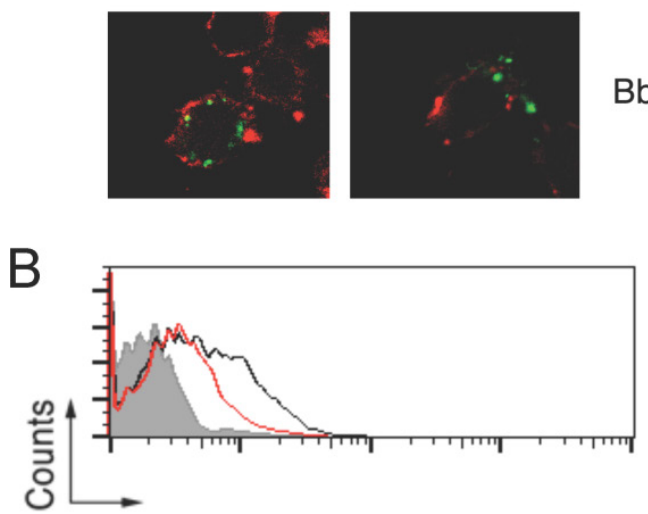

GFP

C

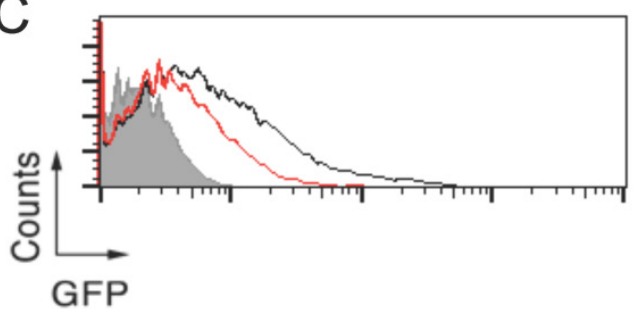

$\mathrm{Bb}$

D $\alpha \mathrm{CD} 16 / \mathrm{CD} 32$

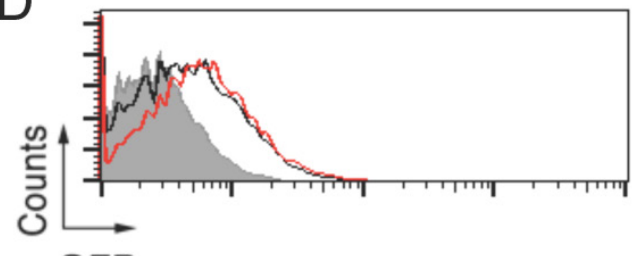

GFP

E

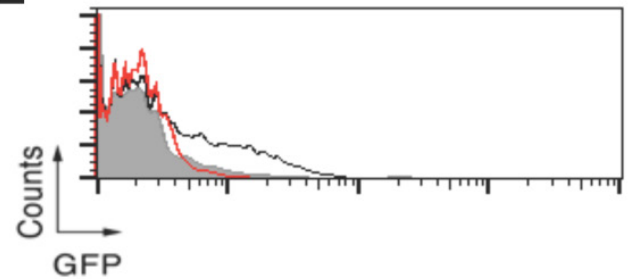

Fig 2. Lipid rafts are required for CR3-dependent, but not CR3-independent, phagocytosis of B. burgdorferi. (A) RAW 264.7 cells were pretreated with I mM of M $\beta C D$ for $30 \mathrm{~min}$ or left untreated, followed by incubation with $10 \mu \mathrm{g} / \mathrm{mL}$ of an anti-CDI6/CD32 mAb and an anti-rat lgG for 30 min (top panels) or Bb9l4 (m.o.i. = 25) for I h (bottom panels). After extensive washing, the cells were fixed and those incubated with $B$. burgdorferi were stained with CTxB Alexa Fluor 594. RAW 264.7 cells (B), WT (C) and CDI I b-deficient BMMs (D) and CHO-CR3/CDI4 cells (E) were pretreated with 5 $\mathrm{mM}$ of $\mathrm{M} \beta C D$ for $30 \mathrm{~min}$ (red histograms) or left untreated (black histograms), followed by incubation with Bb9/4, at the following m.o.i.: RAW264.7 and $\mathrm{CHO}-\mathrm{CR} 3 / \mathrm{CDI} 4,25$; BMMs, 10 . The cells were analyzed by flow cytometry. The grey histogram represents the control performed at $4{ }^{\circ} \mathrm{C}$. The experiments shown are representative of at least 3 performed.

A
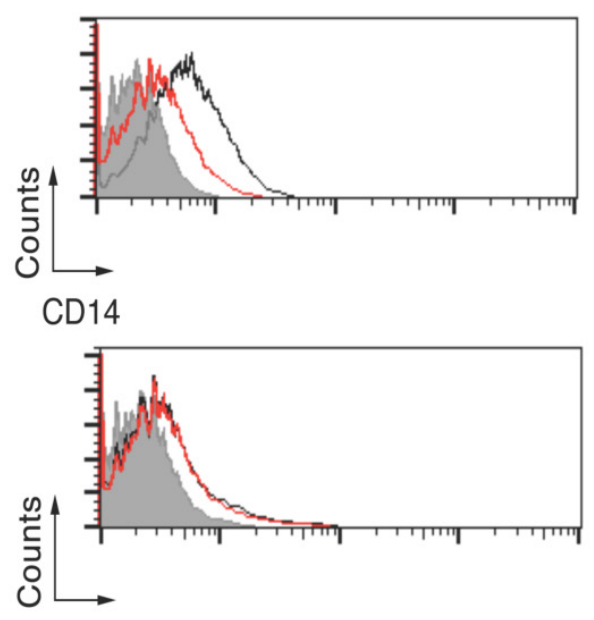

GFP
B
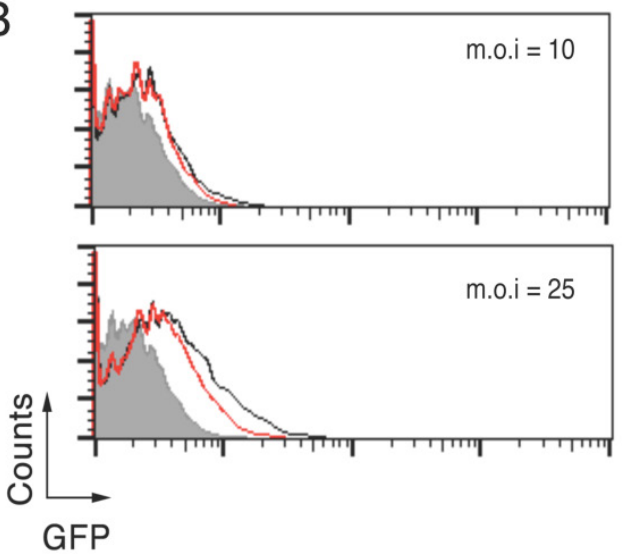

Fig 3. CDI 4 deficiency results in a marginal reduction of $B$. burgdorferi phagocytosis. (A) RAW264.7 cells were treated with I unit of PI-PLC for $20 \mathrm{~min}$ at $4{ }^{\circ} \mathrm{C}$, followed by incubation with Bb9/4 at an m.o.i $=25$. The top panel represents the remaining CDI4 surface expression after PI-PLC treatment (red histogram) compared to untreated cells (black histogram). The grey histogram corresponds to the staining with an isotype control antibody. The bottom panel represents internalization of B. burgdorferi. The grey histogram corresponds to a $4{ }^{\circ} \mathrm{C}$ control. (B) CDI4-deficient (red histograms) or wild type control BMMs (Black histograms) were assessed by flow cytometry for their capacity to phagocytose Bb914 at m.o.i. = 10 (top panel) and 25 (bottom panel). The grey histogram represents the $4{ }^{\circ} \mathrm{C}$ control. The experiments are representative of at least 3 independent experiments. 
Ligation of the C-lectin domain of CR3 triggers B. burgdorferi phagocytosis. The interaction of CD14 and other GPI-anchored proteins with CR3 occurs at the C-lectin domain of the integrin. Phagocytosis of zymosan and other microorganisms can progress in the absence of CD14 (i.e. CHO-CR3 cells [14]). This is likely because zymosan particles bind both the I and the C-lectin domains of the integrin [14], which allows its crosslinking in the absence of other receptors. We argued that the role of CD14 during CR3-mediated phagocytosis of B. burgdorferi consists of its interaction with the C-lectin domain that mimics the effect of zymosan binding to this domain. Glucans are molecules that bind the C-lectin domain of CR3 [13]. Thus, we tested the effect of glucans from different origins on the phagocytosis of B. burgdorferi by RAW264.7 cells. While barley $\beta$-glucan did not have an effect on B. burgdorferi phagocytosis, both laminarin and, to a greater extent, $S$. cerevisiae $\beta$-glucan, increased the capacity of RAW264.7 cells to internalize the spirochete (Figure 4A). Furthermore, coincubation of CHO-CR3 cells lacking CD14 with B. burgdorferi and $S$. cerevisiae $\beta$-glucan resulted in the internalization of the spirochete (Figure 4B), confirming that ligands capable of binding the C-lectin domain of CR3 can induce the crosslinking of the integrin and the efficient internalization of $B$. burgdorferi.

Overall, our results show that the GPI-anchored protein, CD14, interacts with CR3 at the cholesterol-rich membrane microdomains that are essential for the internalization of $B$. burgdorferi mediated by the integrin. These data also reveal that low levels of the CD14 surface pool participate in this process and that crosslinking of CR3 through $\beta$-glucan binding to the C-lectin domain is sufficient to allow the phagocytic process to occur.
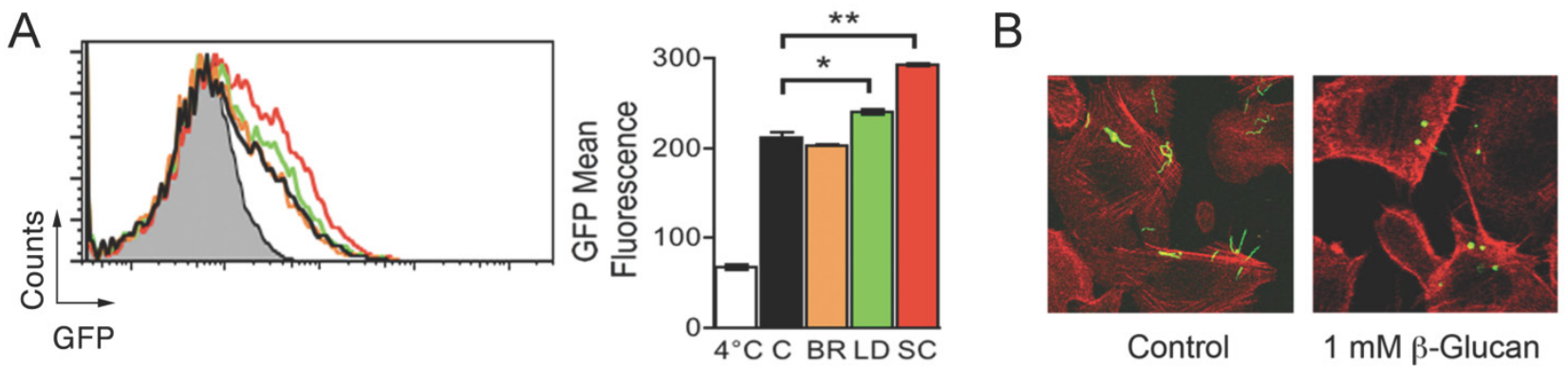

Fig 4. Internalization of B. burgdorferi by CHO-CR3 cells is facilitated by $\beta$-glucans. (A) RAW264.7 cells were incubated with I mM barley $\beta$-glucan (orange histogram), Laminarin $\beta$-glucan (green histogram) or S. cerevisiae $\beta$-glucan (red histogram) and Bb9l4 (m.o.i. $=25)$ for 4 hours. Control cells (black histogram) were incubated with Bb9|4. (B) CHO-CR3 cells were incubated with I mM S. cerevisiae $\beta$-glucan and Bb9l4 for 4 hours. The cells were fixed, stained with phalloidin Alexa Fluor 594 and analyzed by confocal microscopy. The results presented are representative of 3 experiments preformed in triplicate.

\section{Discussion}

Much is still unknown about the specifics of $B$. burgdorferi phagocytosis, despite its importance in the host response during infection, not only related to the elimination of the spirochete, but also to the induction of a proinflammatory deleterious state. The internalization of B. burgdorferi by phagocytic cells is a complex phenomenon that depends on at least 2 receptors that involve very different signaling pathways and results in diverse inflammatory outputs. Thus, both MyD88-dependent and independent phagocytosis occur upon the pathogen interaction with monocytes/macrophages. We have shown that CR3 is a phagocytic receptor that does not require MyD88-derived signals and results in the tempering of the proinflammatory output of these cells [8]. CR3-mediated phagocytosis of B. burgdorferi requires, however, the participation of the GPI-anchored protein, CD14, which does not contribute to the binding to the spirochete [8].

The data presented here indicate that CR3-mediated phagocytosis requires the translocation of the integrin to lipid rafts, which could be mediated by CD14. The requirement of cholesterol during the internalization of the spirochete seems to be independent of the newly described role of this molecule on B. burgdorferi biology [24-26], since the cholesterol-sequestering drug did not affect the uptake of the bacterium in the absence of CD11b. Whether CD14 is unique among GPI-anchored proteins in its ability to promote CR3-dependent phagocytosis of spirochetes remains to be addressed. Sahay and coworkers reported a lack of a noticeable effect in the phagocytic capacity of macrophages that are deficient for CD14 against B. burgdorferi [20]. We analyzed further the 
capacity of CD14-deficient macrophages to internalize the spirochete. As reported by Sahay et al. [20], CD14 null BMMs phagocytosed B. burgdorferi similar to wild type macrophages at an m.o.i of 10. However, at a higher m.o.i $(=25)$ a partial defect in the phagocytic capacity of CD14-deficient BMMs against B. burgdorferi was noted. The reduction in the phagocytic activity of BMMs in the absence of CD14 is much more modest than that observed in CD11b-deficient macrophages. These results suggest the existence of other GPI-anchored proteins that can exert a redundant function during CR3-mediated phagocytosis of $B$. burgdorferi. In fact, CR3-mediated phagocytosis of Mycobacterium kansasii occurs with the participation of several GPI-anchored proteins [27]. The promiscuity shown by CR3 may be also a characteristic in the case of B. burgdorferi phagocytosis, and could explain the modest effect that CD14 deficiency has in spirochetal internalization. Hovius and coworkers [28] have provided indirect evidence suggesting that other GPI-anchored proteins may be aiding CR3 during $B$. burgdorferi phagocytosis. They described that Urokinase Receptor (uPAR, CD87) deficiency impairs $B$. burgdorferi phagocytosis. UPAR is a GPI-anchored protein with known association with CR3. For example, it has been shown to cooperate with CR3 in the phagocytosis of iC3b-opsonized sheep red blood cells [29]. Whether UPAR also cooperates with CR3 during the phagocytosis of the spirochete is worth pursuing and will be the subject of future investigations.

These and our previous results [8] also suggest a direct interaction between CR3 and CD14. GPI-anchored proteins interact with CR3 through the C-lectin domain in the integrin [13]. Thus, we argued that sugars such as lectins, which also bind this domain, could have the capacity to enhance and/or supplement the role played by CD14. Indeed, our results show that the $S$. cerevisiae $\beta$-glucan significantly enhances the internalization of $B$. burgdorferi by RAW cells. Importantly, the use of this glucan allows CHO-CR3 cells, which lack the co-receptor CD14 and are unable to internalize the spirochete [8], to phagocytose B. burgdorferi. These data are in agreement with the capacity of CR3 alone to internalize zymosan particles, which also contain glucan, and provide further evidence that the role played by CD14, likely through its interaction with the C-lectin domain of the integrin, consists on the facilitation of receptor clustering and the initiation of the required signals for the phagocytic process. Overall, our data show evidence of the mechanism at the molecular level of phagocytosis of B. burgdorferi mediated by CR3 in cooperation with CD14.

\section{Acknowledgements}

We are indebted to Justin Radolf for providing the GFP-expressing B. burgdorferi strain and stimulating discussions. We are also grateful to Robin Ingalls for the $\mathrm{CHO}-\mathrm{CR} 3$ cells.

JMIP present address: Institute of Genetic Medicine, Dept. of Molecular Microbiology and Immunology. University of Southern California, Los Angeles, CA 90033.

KLH present address: Department of Pediatrics. University of Connecticut Health Center, Farmington, CT 06030.

\section{Competing Interests}

The authors have declared that no competing interest exists.

\section{References}

1. Greenberg S, Grinstein S. Phagocytosis and innate immunity. Curr Opin Immunol. 2002; 14: 136-45.

2. Moore MW, Cruz AR, Lavake CJ, Marzo AL, Eggers CH, Salazar JC, et al. Phagocytosis of Borrelia burgdorferi and Treponema pallidum potentiates innate immune activation and induces gamma interferon production. Infect Immun. 2007; 75: 2046-62.

3. Shin OS, Isberg RR, Akira S, Uematsu S, Behera AK, Hu LT. Distinct roles for MyD88 and Toll-like receptors 2, 5, and 9 in phagocytosis of Borrelia burgdorferi and cytokine induction. Infect Immun. 2008; 76: 2341-51.

4. Olson C, Bates T, Izadi H, Radolf J, Huber S, Boyson J, et al. Local Production of IFN-gamma by Invariant NKT Cells Modulates Acute Lyme Carditis. Journal of Immunology. 2009; 182: 3728-34.

5. Salazar JC, Duhnam-Ems S, La Vake C, Cruz AR, Moore MW, Caimano MJ, et al. Activation of human monocytes by live Borrelia burgdorferi generates TLR2-dependent and -independent responses which include induction of IFN-beta. PLoS Pathog. 2009; 5: e1000444.

6. Cervantes JL, Dunham-Ems SM, La Vake CJ, Petzke MM, Sahay B, Sellati $\mathrm{TJ}$, et al. Phagosomal signaling by Borrelia burgdorferi in human monocytes involves Toll-like receptor (TLR) 2 and TLR8 cooperativity and TLR8-mediated induction of IFN-beta. Proc Natl Acad Sci U S A. 2011; 108: 3683-8.

7. Cruz AR, Moore MW, La Vake CJ, Eggers CH, Salazar JC, Radolf JD. Phagocytosis of Borrelia burgdorferi, the Lyme disease spirochete, potentiates innate immune activation and induces apoptosis in human monocytes. Infect Immun. 2007; 76: 56-70.

8. Hawley KL, Olson CM, Jr., Iglesias-Pedraz JM, Navasa N, Cervantes JL, Caimano MJ, et al. CD14 cooperates with complement receptor 3 to mediate MyD88-independent phagocytosis of Borrelia burgdorferi. Proc Natl Acad Sci U S A. 2012; 109: 1228-32.

9. Bolz DD, Sundsbak RS, Ma Y, Akira S, Kirschning CJ, Zachary JF, et al. MyD88 plays a unique role in host defense but not arthritis development in Lyme disease. J Immunol. 2004; 173: 2003-10.

10. Liu N, Montgomery RR, Barthold SW, Bockenstedt LK. Myeloid differentiation antigen 88 deficiency impairs pathogen clearance but does not alter inflammation in Borrelia burgdorferi-infected mice. Infect Immun. 2004; 72: 3195-203.

11. Shin OS, Miller LS, Modlin RL, Akira S, Uematsu S, Hu LT. Downstream signals for MyD88-mediated phagocytosis of Borrelia burgdorferi can be initiated by TRIF and are dependent on PI3K. J Immunol. 2009; 183: 491-8.

12. Ueda T, Rieu P, Brayer J, Arnaout MA. Identification of the complement iC3b binding site in the beta 2 integrin CR3 (CD11b/CD18). Proc Natl Acad Sci U S A. 1994; 91: 10680-4

13. Ross GD. Role of the lectin domain of Mac-1/CR3 (CD11b/CD18) in regulating intercellular adhesion. Immunol Res. 2002; 25: 219-27.

14. Le Cabec V, Cols C, Maridonneau-Parini I. Nonopsonic phagocytosis of zymosan and Mycobacterium kansasii by CR3 (CD11b/CD18) involves distinct molecular determinants and is or is not coupled with NADPH oxidase activation. Infect Immun. 2000; 68: 4736-45.

15. Sendide K, Reiner NE, Lee JS, Bourgoin S, Talal A, Hmama Z. Cross-talk between CD14 and complement receptor 3 promotes phagocytosis of 
mycobacteria: regulation by phosphatidylinositol 3-kinase and cytohesin-1. J Immunol. 2005; 174: 4210-9.

16. Kindzelskii AL, Laska ZO, Todd RF, 3rd, Petty HR. Urokinase-type plasminogen activator receptor reversibly dissociates from complement receptor type 3 (alpha $\mathrm{M}$ beta $2^{\prime} \mathrm{CD} 11 \mathrm{~b} / \mathrm{CD} 18$ ) during neutrophil polarization. J Immunol. 1996; 156: 297-309.

17. Zarewych DM, Kindzelskii AL, Todd RF, 3rd, Petty HR. LPS induces CD14 association with complement receptor type 3 , which is reversed by neutrophil adhesion. J Immunol. 1996; 156: 430-3.

18. Dunham-Ems SM, Caimano MJ, Pal U, Wolgemuth $\mathrm{CW}$, Eggers $\mathrm{CH}$, Balic A, et al. Live imaging reveals a biphasic mode of dissemination of Borrelia burgdorferi within ticks. J Clin Invest. 2009; 119: 3652-65.

19. Zhu X, Owen JS, Wilson MD, Li H, Griffiths GL, Thomas MJ, et al. Macrophage ABCA1 reduces MyD88-dependent Toll-like receptor trafficking to lipid rafts by reduction of lipid raft cholesterol. J Lipid Res. 2010; 51: 3196-206.

20. Sahay B, Patsey RL, Eggers CH, Salazar JC, Radolf JD, Sellati TJ. CD14 signaling restrains chronic inflammation through induction of p38-MAPK/SOCS-dependent tolerance. PLoS Pathog. 2009; 5: e1000687.

21. Triantafilou M, Morath S, Mackie A, Hartung T, Triantafilou K. Lateral diffusion of Toll-like receptors reveals that they are transiently confined within lipid rafts on the plasma membrane. J Cell Sci. 2004; 117: 4007-14.

22. Schmitz G, Orso E. CD14 signalling in lipid rafts: new ligands and co-receptors. Curr Opin Lipidol. 2002; 13: 513-21.

23. Bournazos S, Hart SP, Chamberlain LH, Glennie MJ, Dransfield I. Association of FcgammaRIIa (CD32a) with lipid rafts regulates ligand binding activity. J Immunol. 2009; 182: 8026-36.

24. Larocca TJ, Pathak P, Chiantia S, Toledo A, Silvius JR, Benach JL, et al. Proving Lipid Rafts Exist: Membrane Domains in the Prokaryote Borrelia burgdorferi Have the Same Properties as Eukaryotic Lipid Rafts. PLoS Pathog. 2013; 9: e1003353.

25. Crowley JT, Toledo AM, LaRocca TJ, Coleman JL, London E, Benach JL. Lipid exchange between Borrelia burgdorferi and host cells. PLoS Pathog. 2013; 9: e1003109.

26. LaRocca TJ, Crowley JT, Cusack BJ, Pathak P, Benach J, London E, et al. Cholesterol lipids of Borrelia burgdorferi form lipid rafts and are required for the bactericidal activity of a complement-independent antibody. Cell Host \& Microbe. 2010; 8: 331-42.

27. Peyron P, Bordier C, N'Diaye EN, Maridonneau-Parini I. Nonopsonic phagocytosis of Mycobacterium kansasii by human neutrophils depends on cholesterol and is mediated by CR3 associated with glycosylphosphatidylinositol-anchored proteins. J Immunol. 2000; 165: 5186-91.

28. Hovius JW, Bijlsma MF, van der Windt GJ, Wiersinga WJ, Boukens BJ, Coumou J, et al. The urokinase receptor (uPAR) facilitates clearance of Borrelia burgdorferi. PLoS Pathog. 2009; 5: e1000447.

29. Pliyev BK, Arefieva TI, Menshikov MY. Urokinase receptor (uPAR) regulates complement receptor 3 (CR3)-mediated neutrophil phagocytosis. Biochem Biophys Res Commun. 2010; 397: 277-82. 